\title{
Project manager of integrated report: una experiencia de formación en el contexto del informe de sostenibilidad
}

\author{
PATRICE DE MICCO ${ }^{1}$; ERINA GURAZIU²; RODRIGO LATORRE ${ }^{3}$
}

\section{RESUMEN}

La investigación analiza el programa formativo del Proyecto PMIR (Project Manager of Sustainability Report/Integrated Report), cuyo objetivo es crear una figura profesional capaz de coordinar la elaboración de Informes de Sostenibilidad, proporcionando información económica, social y ambiental de empresas de cualquier tipo.

Objetivo. Nuestro estudio contribuye al debate sobre la educación en sostenibilidad al examinar cómo los programas educativos pueden y deben responder a la creciente complejidad del Desarrollo Sostenible.

Metodología. La investigación adoptó un enfoque cuantitativo con alcance descriptivo y diseño exploratorio. Se presenta una encuesta realizada en Italia, Alemania, España, Bélgica y Chile, a través de cuestionarios a empresas con y sin experiencia en informes de sostenibilidad.

Resultados. Con respecto a las competencias que faltan en las empresas europeas, en general, para elaborar los Informes de Sostenibilidad, el $21 \%$ son relacionadas con la estadística, el $18 \%$ económicas y $17 \%$ relacionales. En las empresas chilenas, las habilidades necesarias a mejorar son de igual naturaleza.

Discusión. La sostenibilidad requiere un enfoque transdisciplinario y transnacional, requeriendo adaptar la educación, debido a su naturaleza global.

Conclusiones. Es esencial adoptar un marco común para definir competencias, habilidades y conocimientos, tal como la metodología ECVET (European Credit System for Vocational Education and Training), que permite un alcance transdisciplinar e internacional en reconocer las competencias.

Palabras claves: Educación en Sostenibilidad, Transdisciplinariedad, Informe de Sostenibilidad, Resultados de Aprendizaje, Gestión de Proyectos

\section{Project manager of integrated report: a training experience in the context of sustainability reporting}

\begin{abstract}
This research analyses the training experience of the PMIR Project, whose main objective is the creation of a professional figure capable of coordinating the preparation of Sustainability Reports, within companies of any type. The Sustainability Report is a complex process that aims to provide stakeholders with relevant information about the economic, social and environmental performance of an organization. Our study contributes to the debate on sustainability education by examining a case study of how educational programs can and should respond to the growing complexity and needs of the current economic context. We showed, through the analysis of a survey conducted in Italy, Germany, Spain, Belgium and Chile, among companies with experience in sustainability reporting, that sustainability requires the integration of different disciplines, so training and education is needed to adopt a transdisciplinary approach. In addition, the educational approach must also be transnational since the skills and competences developed must be easily recognised in different countries due to the global nature of sustainability reporting. In this regard, it is essential to adopt a common framework to define competences, skills and knowledge, such as the ECVET methodology that was used to define the objectives and content of the PMIR training program.
\end{abstract}

Keywords: Sustainability Education, Transdisciplinarity, Sustainability Reporting, Learning Outcomes, Project Management 


\section{INTRODUCCIÓN}

El objetivo general del artículo es demostrar a través de un ejemplo concreto, como la situación económica y social de hoy, complicada y dinámica, es capaz de crear nuevas oportunidades de trabajo y requiere un papel activo y participativo por parte de la formación, con respecto a la introducción de nuevas competencias, capacidades y conocimientos. Un ejemplo válido, por cierto, es el ámbito de Sostenibilidad. Para alcanzar el objetivo planteado, se presenta y analiza el programa formativo del proyecto PMIR (Project Manager of Sustainability Report/Integrated Report), como experiencia transdisciplinar y transnacional en Europa que permite comprender fenómenos contemporáneos complejos, desde una perspectiva holística, y su posible efecto en la Empleabilidad.

Por lo tanto, se evidencia la "Transdisciplina" en la Formación, como requisito para comprender fenómenos contemporáneos complejos, asumiendo finalmente, un marco de referencia compartido, (ECVET), para describir conocimientos, competencias y capacidades, facilitando la empleabilidad más allá de las fronteras nacionales y creando un lenguaje común para evaluar los perfiles profesionales.

EI Proyecto PMIR (Project Manager of Sustainability Report/Integrated Report) fue financiado en el 2017 en Italia por el Programa Europeo Erasmus+, tarda 3 años y está actualmente en desarrollo. El objetivo es crear un perfil profesional responsable del proceso de elaboración del Informe de Sostenibilidad/ Informe Integrado dentro de una empresa de cualquier tamaño. Esta iniciativa, permite apreciar el aporte que la educación y la formación proporcionan a los individuos para enfrentar y explotar tanto los desafíos como las oportunidades del contexto económico y social contemporáneo. Es una respuesta a la Directiva 2014/95/UE que, desde 2017, solicita a ciertas empresas europeas, presentar un Informe anual de su gestión no financiera. Además de cumplir con la legislación, el proyecto entrega las herramientas que permiten a las empresas dar a conocer su compromiso con la Sostenibilidad, a través de un informe que refleja una visión transdisciplinaria de su gestión, integrando objetivos económicos, ambientales y sociales.

Para conseguir sus objetivos transdisciplinares, el consorcio del proyecto fue compuesto para representar las competencias pluridisciplinarias necesarias, incluyendo las de: gran empresa, universidad (Facultad de Ciencias de Educación), empresa social (Italia), cámaras de comercio
(Alemania y España), expertos en la metodología ECVET (Bélgica y Noruega). El resultado final es una figura profesional innovadora "Director de Proyecto de Redacción del Informe de Sostenibilidad/Informe Integrado" - PMIR.

El proyecto PMIR incluye: investigación y desarrollo de una plataforma educativa (Año 1); matriz de resultados de aprendizaje (PMIR) y formación en línea (Año 2); Plataforma de Base de Datos para el Informe Integrado, prueba piloto y movilidad europea de los participantes (Año 3). Además, el proyecto considera un pilotaje sobre la transferencia de dichas competencias a otras regiones mundiales, como la Alianza del Pacifico, empezando por Chile, líder de la región en términos de desarrollo económico, social e institucional, que enfrenta desafíos de desigualdades y Sostenibilidad económica.

En el contexto de la Unión Europea, donde la movilidad de los individuos es uno de los principios fundantes, a partir del 2004, con la Maastricht Communiqué, la Comisión Europea fortaleció su papel en el reconocimiento entre países, de competencias innovadoras que responden a necesidades del mercado de trabajo (Sinyagovskaya, 2018). Esta iniciativa permitió introducir las herramientas que facilitan el dialogo entre los Marcos Nacionales de Cualificaciones, uno de las cuales es ECVET (Recomendación 2009/C 155/02, 2009) que tiene como objetivo el desarrollo de cualificaciones mediante resultados de aprendizaje, permitiendo la validación, reconocimiento y certificación de competencias entre Países Miembros de la Unión Europea, facilitando así movilidad, empleabilidad e inclusión laboral de las personas.

El desafío del proyecto era reconocer los resultados de aprendizaje por los menos en los 5 países del proyecto (Italia, España, Alemania, Noruega, Bélgica) para facilitar a los alumnos trabajar en otro país europeo, una vez cualificados. Por esto, se utilizó el marco ECVET, que describe las cualificaciones en unidades, con resultados de aprendizaje. ECVET permite la comunicación entre educación formal (secundaria y superior), no formal e informal, contribuyendo eficazmente al reconocimiento de aprendizaje previo u experiencial, de conformidad con las normas y prácticas de los países participantes, lo que facilita la movilidad transnacional, pero también horizontal y vertical entre sectores y contextos de aprendizaje (formal, no formal e informal) y finalmente entre sistemas (educación superior, educación y formación profesional), soportando la empleabilidad de los individuos.

Artículo 3, apartado 2, del Tratado de la Unión Europea (TUE); artículo 4, apartado 2, letra a) y artículos 20, 26 y 45 a 48 del Tratado de Funcionamiento de la Unión Europea (TFUE)

European Credit for Vocational Education and Training.

Un marco técnico para la transferencia, el reconocimiento y, cuando proceda, la acumulación de resultados individuales de aprendizaje con vistas a obtener una cualificación (Recomendación 2009/C 155/02,2009)

Conocimiento: resultado de asimilación de información gracias al aprendizaje; Capacitades: habilidad para aplicar conocimiento y utilizar técnicas a fin de completar tareas y resolver problemas; Competencias: capacitad demostrada para utilizar conocimiento, destreza y habilidades personals, sociales y metodológicas, en situaciones de trabajo o estudio y en el desarrollo profesional y personal. (Recomendación 2017/C 189/03) 
El proyecto, se enmarca en el concepto de Sostenibilidad como se conoce actualmente, que considera el concepto de "Sociedad Sustentable" y su representación, en el centro de la intersección de tres círculos, (ámbitos económico, ambiental y social), ampliamente difundida (Purvis, et al., 2018), constituyendo un desafío a la empresa para crear valor de largo plazo para sus grupos de interés, centrándose en las dimensiones sociales, económicas y ambientales de las organizaciones empresariales (Ashrafi et al., 2019). Por lo anterior, la responsabilidad empresarial necesita de un enfoque definido para integrarla dentro de la estrategia y de los procesos empresariales (Baumgartner and Ebner, 2010), asumiendo que es un concepto complejo y cambiante que incluye muchos elementos y actores diferentes (Baret and Helfrich, 2018; Schaltegger et al., 2013), lo que ha demandado cambios en las prácticas contables, para satisfacer la presión informativa de los stakeholders (Gray, 2001; Bebbington and Larrinaga, 2014) incrementando la calidad de la información no financiera (loannou and Serafeim, 2017).

La naturaleza multidimensional que caracteriza el informe de Sostenibilidad y el importante papel que este juega dentro de la responsabilidad empresarial instan a adoptar con urgencia, competencias relacionadas con la Sostenibilidad $y$, por lo tanto, piden un papel activo a la educación y formación, en este sentido Dale and Newman (2005), reconocen que la educación en desarrollo sustentable necesita ser compleja, transdisciplinaria y amplia, surgiendo iniciativas como el juego de simulación de negocios planteado por Baumgartner and Winter (2013), para formar en línea a los directores de Sostenibilidad, con un enfoque multidisciplinario. La necesidad que la sostenibilidad en general requiere de un enfoque multidisciplinario es confirmada también por Wamsler (2020), quien afirma que la planeación actual de la educación en sostenibilidad debería adoptar modalidades más integrales. La formación y la educación requieren una conexión permanente con el mundo de trabajo, para constituir una respuesta actualizada a los fenómenos complejos que caracterizan el mercado laboral contemporáneo, y que demandan una renovación continua de las competencias, como se establece también en el Future of Jobs Report (World Economic Forum, 2016)

Este artículo contribuye a este debate mediante la investigación de las competencias interdisciplinarias necesarias para la elaboración del informe de sostenibilidad y la descripción de un resultado concreto como es el programa formativo no formal PMIR que permite el reconocimiento internacional formal de dichas competencias desarrolladas en resultados de aprendizaje.

\section{MÉTODOS}

Se presenta el proyecto que ha permitido desarrollar a nivel europeo estas competencias transdisciplinares y transnacionales en el marco de la gestión de un Informe de Sostenibilidad dentro de una organización. Se presentará la metodología de investigación de competencias, la prospectiva de transferirlas a un país de América Latina, Chile, y el marco de planeación formativa por resultados de aprendizaje, ECVET, que permite experimentar esta traslación transcontinental.

El objetivo es investigar las competencias necesarias para desarrollar el informe de sostenibilidad, siguiendo un enfoque bottom-up de descripción de resultados de aprendizaje, mediante la involucración de las empresas con experiencia en la elaboración del informe. La investigación se realizó adoptando un enfoque cuantitativo con un alcance descriptivo y diseño exploratorio, empleando como instrumento de recolección de datos mediante cuestionarios con preguntas cerradas.

Esta investigación se desarrolló durante el primer año del Proyecto PMIR, con el propósito de entender la percepción de la importancia social del tema de la sostenibilidad e identificar las áreas educativas a incluir en las unidades que componen el currículum del perfil profesional PMIR. La investigación se ha enfocado en las siguientes fases: 1 . revisión bibliográfica (publicaciones desde 2001 hasta 2007) sobre la relación entre Sostenibilidad y empresas, estableciendo la evolución del concepto de Sostenibilidad; 2. comprensión de los cambios organizativos de las empresas para alcanzar niveles de Sostenibilidad más altos, así como la manera en que las empresas exponen sus impactos ambientales y sociales; 3 . administración de tres cuestionarios en Bélgica, Alemania, Italia y España, dirigidos a consumidores (I cuestionario), empresas de cualquier tipo (II cuestionario) y empresas con experiencia en la elaboración de Informes de Sostenibilidad (III cuestionario); 4. interpretación de los datos y elaboración de las conclusiones sobre las competencias a trasferir al Project Manager of Integrated Report/Sustainability Report (PMIR).

En particular, el segundo cuestionario, dirigido a empresas de cualquier tipo, constó de ocho preguntas que tenian como propósito evidenciar el nivel de conocimiento del concepto de sostenibilidad en las empresas. Además, destacó la relevancia presente y futura que este concepto asume para las empresas, investigando las prioridades empresariales y el papel que la sostenibilidad juega dentro de ellas. También investigó la posible experiencia en el reporte de sostenibilidad de estas empresas.

El tercer cuestionario constó de once preguntas relativas a la experiencia de las empresas en 
Informes de Sostenibilidad, las razones que las impulsaron a llevar a cabo este proceso, los beneficios esperados y realizados, así como las dificultades encontradas y las habilidades y competencias consideradas necesarias. El tercer cuestionario, entonces, permitió identificar las competencias que, para las empresas, debe tener un perfil profesional que coordina el proceso de elaboración de los Informes de Sostenibilidad. En Europa, con el objetivo de obtener una muestra representativa del tejido productivo europeo, el cuestionario fue administrado a 76 empresas (21 en Bélgica, 16 en Alemania, 19 en Italia y 20 en España), que pertenecieron a sectores diferentes; en particular, el 27,6\% al sector industrial, el 10,5\% al comercio, y el $7 \%$ al sector de transportes y servicios. La muestra se constituyó sobre todo por empresas grandes y medianas, aunque en España se notó una presencia consistente de microempresas.

La administración de los cuestionarios dos y tres se ha repetido en Chile, involucrando empresas chilenas con y sin experiencia en la elaboración de informes de sostenibilidad, con el fin de comprender cuales son las competencias requeridas.

Con respecto al contexto chileno, se debe destacar que desde una perspectiva pública, el Consejo de Desarrollo Sustentable de Chile actúa, desde 1998, como un organismo asesor de la Presidencia de la República, constituyendo la instancia de mayor participación ciudadana e institucional del país, de diversos estamentos sociales en los procesos de toma de decisión relacionados al Desarrollo Sustentable. Desde una perspectiva privada, desde el 2006, se elaboran Reportes de Sostenibilidad, esencialmente, por grandes empresas, cuya publicación en la plataforma del GRI en 2017, presenta 34 informes.

Las empresas encuestadas en Chile, pertenecieron a ASIVA, asociación gremial de grandes, medianas y pequeñas empresas de los sectores industriales, productivos y de servicios de la Región de Valparaíso. En esta primera investigación, se recibieron las respuestas de nueve empresas encuestadas, tres de ellas con experiencia en la elaboración de Informes de Sostenibilidad y seis de ellas, sin experiencia. La muestra chilena, en comparación con la europea, está representada por un un menor número de empresas, porqué, esencialmente, son menos aquellas que actualmente se dedican a la presentación de informes de sostenibilidad/informes integrados en Chile.

Entre las empresas con experiencia, dos de ellas son grandes empresas y una mediana. Una de ellas pertenece al sector Servicios Portuarios, otra a Energía y la tercera a Servicios Básicos.

En el caso de aquellas empresas sin experiencia en la elaboración del informe, la muestra incluyó a organizaciones del sector de Educación Superior, Embotelladoras, Servicio de Seguros y Servicio de Consultoría.

\section{RESULTADOS}

Como se mencionó anteriormente, se aplicaron dos encuestas en Bélgica, Alemania, Italia, España y Chile a empresas de diversos tamaños, que operan en diferentes sectores con y $\sin$ experiencia en informes de sostenibilidad.

En cuanto a la muestra europea, y en relación a las competencias necesarias para implementar y llevar a cabo con éxito los Informes de Sostenibilidad, se solicitó que las empresas identificaran aquellas más relevantes, como también aquellas más carentes para la elaboración del informe. El resultado de la investigación, se muestra en las Figuras 1 y 2.

En ellas, es posible observar que el $30 \%$ de las empresas perciben las habilidades económicas y empresariales como las más relevantes para los informes de sostenibilidad, seguidas de las relacionales $(25 \%)$ y estadísticas $(10,5 \%)$, con la excepción de Italia, en donde las competencias relacionales y de gestión alcanzan el porcentaje mas alto (37\%). Con respecto a las competencias que faltan, en general, el $21 \%$ de las empresas muestra la falta de aquellas estadísticas, el $18 \%$ de aquellas económicas y el $17 \%$ de capacidades relacionales. 


\section{Metodología de investigación de competencias investigación en Europa}

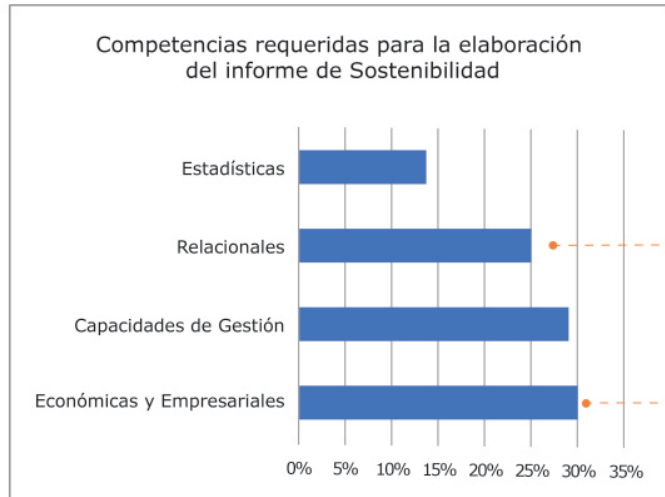

Figura 1.Competencias requeridas para la elaboración del Informe de Sostenibilidad en organizaciones europeas.

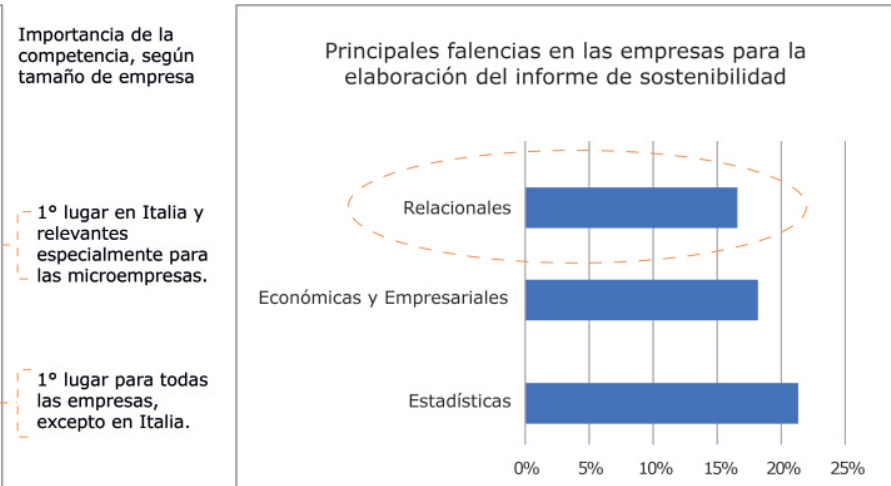

Figura 2. Carencias en las capacidades para la elaboración del Informe de Sostenibilidad en organizaciones europeas.

En las empresas chilenas, las habilidades prioritarias que debería poseer el directivo responsable de la elaboración del informe son las económicas y empresariales y también las blandas. Aquellas a mejorar son las estadísticas, empresariales, relacionales y para establecer metas conjuntas entre distintos departamentos. Los resultados se muestran en las Figuras 3 y 4.

Las organizaciones chilenas coinciden en identificar a las habilidades organizativas y de relación como las más relevantes para la elaboración del informe, proyectando a mediano plazo, el aumento de la importancia de objetivos de Sostenibilidad al mismo nivel que aquellos económicos y comerciales.

\section{Metodología de investigación de competencias investigación en Chile}

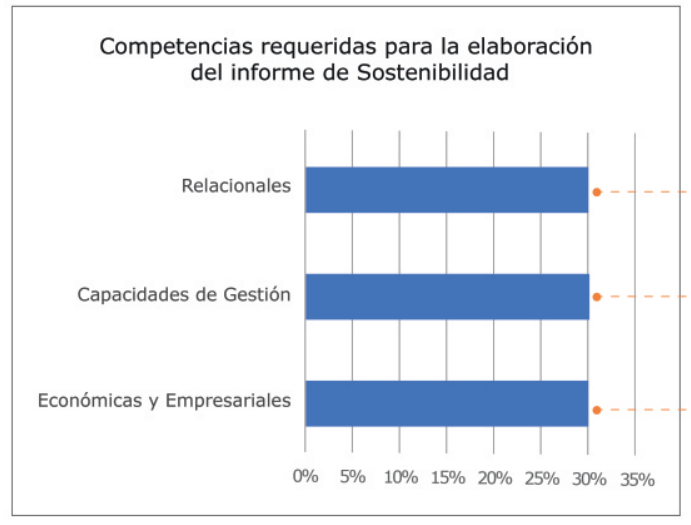

Figura 3.Competencias requeridas para la elaboración del Informe de Sostenibilidad en organizaciones chilenas.

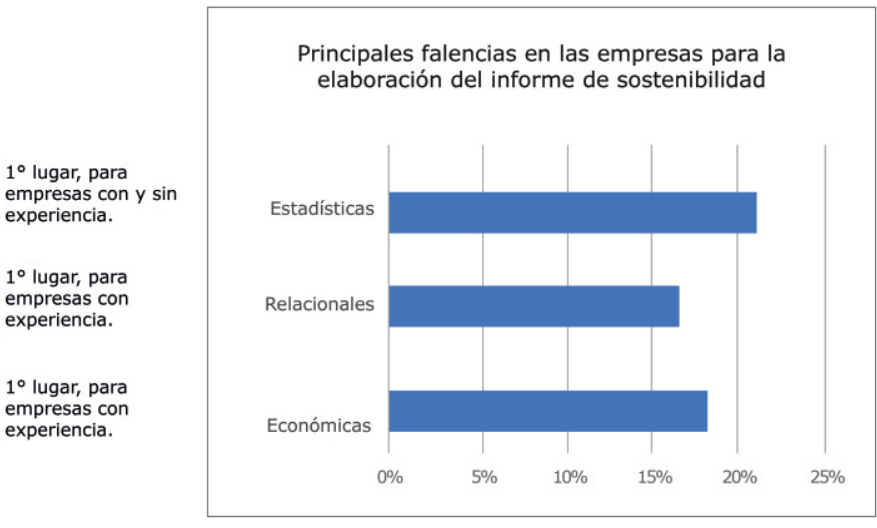

Figura 4. Carencias en las capacidades para la elaboración del Informe de Sostenibilidad en organizaciones chilenas.

Consecuentemente, las empresas en Europa y en Chile se muestran conscientes de la complejidad del proceso de elaboración de informes de Sostenibilidad, que requiere varias competencias de distinta naturaleza: junto a aquellas económicas y comerciales tradicionales, aparecen como las más importantes, las capacidades relacionales, de organización y estadísticas. La diversidad de las competencias requeridas, demanda que su adquisición y transferencia sea articulada en un conjunto coherente de contenidos con un objetivo común. Esto confirma que la forma más eficiente y eficaz de comunicar los alcances de los Informes de Sostenibilidad para la gestión de la empresa, es a través de procesos formativos, transdisciplinares y formalmente estructurados, con un reconocimiento académico oficial, como es el programa PMIR.

Como respuesta a los resultados conseguidos en Europa, la cualificación PMIR se ha desarrollado en una matriz de siete unidades agregadas de manera coherente en resultados de aprendizaje en el nivel EQF entre 7 y 8: U1: Responsabilidad Social Corporativa (CSR); U2: Marco Regulados de información no financiera; U3: Gestión de Datos (Estadísticas y TIC); U4: Gestión de Proyectos; U5: Comunicación; U6: Competencias sociales; U7: Informe de Sostenibilidad e Informe Integrado (áreas económicas, sociales y ambientales). 
Para garantizar la realización de un currículum transnacional, el Proyecto PMIR ha adoptado la metodología ECVET para definir conocimientos, competencias y capacidades, descritos como resultados de aprendizaje, que se pueden transferir, evaluar y reconocer en distintos países. El director PMIR es una figura profesional que posee conocimientos sobre los aspectos de gestión de la sostenibilidad, así como del proceso que la empresa necesita emprender para crear y comunicar valor de manera adecuada para su competitividad.

En conclusión, los resultados de los cuestionarios administrados a las empresas en Europa y en Chile confirman que el perfil profesional creado por el Proyecto PMIR es capaz der responder a solicitudes y necesidades concretas de aquellas empresas que, operando en dos regiones distintas del mundo participan activamente a través de su gestión al Desarrollo Sostenible..

\section{DISCUSIÓN}

EI PMIR nace de la legislación que obliga a las grandes organizaciones a proveer información no financiera a sus grupos de interés. La articulación de contenidos y metodologías del programa, es la respuesta de un programa educativo no formal, a un fenómeno complejo, la Sostenibilidad, relevando dos de sus atributos esenciales: su naturaleza transdisciplinar y su carácter transnacional, aspectos que sólo tienen sentido en una propuesta formativa en el marco de la complejidad contemporánea y que hacen de PMIR una experiencia inédita e innovadora.

Por esto, los resultados de la investigación han permitido hacer una primera comparación entre las competencias requeridas por las empresas europeas y las empresas chilenas. A pesar de la muestra limitada de empresas chilenas y de las deferencias que caracterizan el contexto legal europeo con el chileno, esencialmente por la Directiva Europea que pide una mayor aprobación legislativa, los resultados alcanzados son similares. De hecho, en ambos contextos las competencias consideradas más relevantes para la implementación del informe de sostenibilidad están relacionadas con habilidades de gestión, blandas y estadística, incluyendo también las competencias económicas, requeridas para elaborar y comunicar información de la gestión no financiera de las organizaciones en dos contextos económicos distintos, pero con aspectos comunes en el marco de la Sostenibilidad.

El proyecto PMIR, mediante la investigación y los resultados establecidos en la cualificación del Director, muestra que la Sostenibilidad es un proceso que puede ser manejado mediante competencias transdisciplinares, desarrolladas en contextos internacionales que no reconocen fronteras. Por esto, una formación desarrollada en ambientes educativos no formales, mediante un enfoque bottom-up, que surge de las exigencias de las empresas mismas, puede ser capaz de llevar innovación a la formación formal, fortaleciendo procesos de reconocimiento de competencias entre países.
De hecho, al final de los primeros dos años de proyecto, se ha obtenido el reconocimiento oficial del programa formativo PMIR por parte de la educación superior, atribuyéndole créditos universitarios. La primera formación reconocida se realizó en octubre 2019, solicitando una participación internacional. Para asegurar que los participantes tuvieran los conocimientos y habilidades necesarios para alcanzar el alto nivel del curso (EQF entre 7 y 8 ), se solicitó que demostraran mediante currículum, experiencias desarrolladas en contextos no formales e informales, además de eventuales certificados universitarios.

Lo anteriormente descrito, representa un beneficio de alto valor, tanto para la empleabilidad de los egresados, como para las personas en proceso de reconversión profesional. Además, el reconocimiento de los créditos universitarios permite a los estudiantes universitarios acceder a conocimientos transdisciplinares que difícilmente se podrían adquirir al interior dentro de la institución en dónde cursan sus estudios. Esto conlleva también un beneficio para la educación superior, que puede ofrecer una formación, no solo enfocada en conocimientos específicos académicos, sino abierta a nuevos saberes que nacen desde un contexto empresarial y que responden a resultados de aprendizaje concretos descritos según un enfoque bottom-up. De esta forma, el proyecto ha permitido conectar educación superior, con la educación y formación profesional, contexto empresarial y políticas de gobierno (Directiva 2014/95/UE, 2014), fortaleciendo un proceso conjunto que tiene como objetivo, la empleabilidad de los egresados y personas en el mundo del trabajo dentro de un contexto mundial actual en rápido cambio.

${ }^{7}$ Nivel 7: conocimiento y capacidades altamente especializadas; gestión y transformación de contextos; Nivel 8: conocimientos en la frontera más avanzada de un campo de trabajo; autoridad, innovación, autonomía. 


\section{CONCLUSIONES}

El proyecto PMIR fue elegido como caso de estudio, considerando que su currículum demanda conocimientos, competencias y capacidades transdisciplinares para la elaboración del Informe de Sostenibilidad, que pide la integración de disciplinas diferentes, incluyendo economía, comunicación, habilidades blandas y gestión de datos.

Los cuestionarios administrados en los países europeos (Bélgica, Alemania, Italia y España) y en Chile muestran como las empresas consideran las competencias de gestión, blandas y estadísticas como las más importantes para la implementación del proceso de elaboración del Informe de Sostenibilidad.

Para garantizar la realización de un currículum transnacional, el Proyecto PMIR ha adoptado la metodología ECVET para definir conocimientos, competencias y capacidades de cualquier tema de formación. Dicha metodología, de hecho, representa un "lenguaje" claro y compartido para expresar resultados de aprendizaje que el Programa quiere alcanzar.
Una formación trandisciplinaria y transnacional es, simultáneamente, lo que se necesita en el mercado laboral actual, siempre más global y dinámico y a la vez contribuir a la empleabilidad y al crecimiento profesional.

El análisis presentado en este artículo es de tipo cualitativo. Transcurrido un año desde el término del Proyecto, será posible elaborar un estudio de naturaleza cuantitativa, que permita verificar el impacto laboral y de crecimiento que ha representado para los participantes en los diferentes países, y al mismo tiempo, conocer si sus expectativas se han cumplido. De hecho, la participación en el programa PMIR, requirió que los candidatos enviaran su currículum y una carta de motivación, expresando las razones de su postulación, así como sus aspiraciones. Finalmente, el proyecto y la actividad de investigación se podrán extender también a otros países.

Para concluir, la formación está llamada a ser dinámica, interdisciplinaria y a mirar más allá de los límites nacionales, para constituir un instrumento claro en favor de la empleabilidad y el desarrollo del mercado laboral.

\section{REFERENCIAS BIBLIOGRÁFICAS}

Amini M. and Bienstock C. C. (2014). Corporate sustainability: an integrative definition and framework to evaluate corporate practice and guide academic research. Journal of Cleaner Production, 76, 12-19.

Baret, P. and Helfrich, V. (2018). The "trilemma" of non-financial reporting and its pitfalls. Journal of Management and Governance, 1-27.

Baumgartner R. J. and Winter T. (2013). The Sustainability Manager: A Tool for Education and Training on Sustainability Management. Corporate Social Responsibility and Environmental Management, 21, 167-174.

Bengtsson, S. L. and Östman, L. O. (2013). Globalisation and education for sustainable development: Emancipation from context and meaning. Environmental Education Research, 19(4), 477-498.

Bebbington J. and Larrinaga C. (2014). Accounting and sustainable development: An exploration. Accounting, Organizations and Society, 39, 395-413.

Carroll A. B. (1999). Corporate Social Responsibility Evolution of a Definitional
Construct. Business \& Society, 38 (3), 268295.

Dale, A. and Newman, L. (2005), Sustainable development, education and literacy, International Journal of Sustainability in Higher Education, 6(4), 351-362.

Directiva 2014/95/UE. (2014). Parlamento Europeo y el Consejo. Obtenido de eur-lex: https: / / eur-lex.europa.eu/legalcontent/ES/TXT/?uri=CELEX\%3A32014L0 095.

Dyllick T. and Muff K. (2015). Clarifying the Meaning of Sustainable Business: Introducing a Typology from Businessas-Usual to True Business Sustainability. Organization \& Environment, 29(2), 156-174.

González-Gaudiano, E. J. (2005). Education for sustainable development. Policy Futures in Education, 3(3), 243-250.

Gray R. (2001). Thirty years of social accounting, reporting and auditing: what (if anything) have we learnt? Business Ethics - A European Review, 10(1), 9-15. 
Hahn T., Figge F., Pinkse J. and Preuss L. (2010). Trade-Offs in Corporate Sustainability: You Can't Have Your Cake and Eat It. Business Strategy and the Environment. 19. 217 - 229. 10.1002/bse.674.

Hahn T. and Pinkse J. (2015). Tensions in Corporate Sustainability: Towards an Integrative Framework, Journal of Business Ethics, 127(2), 297-316.

Herzig C., Schaltegger S. (2006). Corporate Sustainability Reporting. An Overview. In: Schaltegger S., Bennett M., Burritt R. (eds) Sustainability Accounting and Reporting, Springer.

Jickling B., Sterling S. eds. (2017). PostSustainability and Environmental Education Remaking Education for the Future Foreword, Palgrave Studies in Education and the Environment.

Jones P., Selby D. and Sterling S. eds. (2010). Sustainability Education: Perspectives and Practice across Higher Education, Earthscan.

Martinez Miguelez, M. (2012). Conceptualización de la transdisciplinariedad. Obtenido de Polis Revista Latinoamericana: http://journals.openedition.org/ polis/4623.

Moore J. (2005). Barriers and pathways to creating sustainability education programs: policy, rhetoric and reality, Environmental Education Research, 11(5), 537-555.

PMIR. (2018). Sostentabilidad, Empresas y Consumidores. Informe de Analisis de Necesitades. Obtenido de PMIR: http://www.pmir.it/index.php/projectoutputs/?lang=es.
Purvis, B., Mao, Y. and Robinson, D. (2018). Three pillars of sustainability: in search of conceptual origins. Obtenido de I i n k. s p r i n g e r. c o m : https://link.springer.com/article/10.1007 \%2Fs11625-018-0627-5.

Recomendación 2009/C 155/02. (2009). Parlamento Europeo y Consejo. Obtenido de Eur-Iex: https: / / eurI ex. e u ropa. e u / I e ga I content/ES/TXT/HTML/?uri=CELEX:3200 9H0708(02)\&from=it. Recomendación 2017/C 189/03. (s.f.). Consejo de la Unión Europea. Obtenido de eur-lex: https://eur-lex.europa.eu/legalcontent/ES/TXT/HTML/?uri=CELEX:3201 7H0615(01)\&from=EN.

Schaltegger S. and Burritt R. L. (2010). Sustainability accounting for companies: Catchphrase or decision support for business leaders? Journal of World Business, 45(4), 375-384.\}

Sinyagovskaya, M. (2018). The Turin Process and the Development of Qualifications Frameworks for Working Specialties in Russia and European Countries, https://doi.org/10.2991/iceder18.2018.26.

Wiek, A., Withycombe, L. and Redman, C.L. (2011). Key competencies in sustainability: a reference framework for academic program development. Sustainable Science, 6(2) 203-218.

World Economic Forum. (2016). Future of Jobs Report. Obtenido de Future of Jobs R $\quad$ e $\quad p \quad l \quad r \quad t$, http://reports.weforum.org/future-ofjobs-2016/.

Recibido: 18 marzo 2019 | Aceptado: 20 julio 2019 